\title{
Pratiques
}

Linguistique, littérature, didactique

183-184| 2019

oralité, littératie

\section{Paul et Virginie (1789) ou la fatale irruption de l'écrit}

Paul et Virginie (1789) or the fatal irruption of writing

Jean-Marie Privat

\section{(2) OpenEdition}

Journals

Édition électronique

URL : http://journals.openedition.org/pratiques/7342

DOI : 10.4000/pratiques.7342

ISSN : 2425-2042

\section{Éditeur}

Centre de recherche sur les médiations (CREM)

\section{Référence électronique}

Jean-Marie Privat, «Paul et Virginie (1789) ou la fatale irruption de l'écrit », Pratiques [En ligne], 183-184 | 2019, mis en ligne le 30 décembre 2019, consulté le 10 octobre 2020. URL : http:// journals.openedition.org/pratiques/7342 ; DOI : https://doi.org/10.4000/pratiques.7342

Ce document a été généré automatiquement le 10 octobre 2020

(c) Tous droits réservés 


\title{
Paul et Virginie (1789) ou la fatale irruption de l'écrit
}

\author{
Paul et Virginie (1789) or the fatal irruption of writing
}

Jean-Marie Privat

1 Le roman de Bernardin de Saint-Pierre a hissé ses deux jeunes protagonistes au rang de mythe littéraire :

\begin{abstract}
Avant qu'elle se mariât, elle avait cru avoir de l'amour [...]. Et Emma cherchait à savoir ce que l'on entendait au juste dans la vie par les mots de félicité, de passion et d'ivresse, qui lui avaient paru si beaux dans les livres. Elle avait lu Paul et Virginie et elle avait rêvé la maisonnette de bambous, le nègre Domingo, le chien Fidèle, mais surtout l'amitié douce de quelque bon petit frère, qui va chercher pour vous des fruits rouges dans des grands arbres plus hauts que des clochers, ou qui court pieds nus sur le sable, vous apportant un nid d'oiseau. Lorsqu'elle eut treize ans, son père

l'amena lui-même à la ville, pour la mettre au couvent. (Flaubert, 1971 [1857], p. 36)
\end{abstract}

2 Emma, lectrice, rêve d'une petite société où tout ne serait que don et contre-don, vie harmonieuse avec une nature généreuse, plaisirs du corps et chaleur des relations interpersonnelles, saveurs de la jeunesse et savoirs pratiques du monde. Cette idylle romantique et ce meilleur des mondes lointains (même les serviteurs noirs sont bons et serviables) tiennent sans doute une part de leurs charmes à l'exotisme géographique tel que revendiqué par l'écrivain :

Nos poètes ont assez reposé leurs amants sur le bord des ruisseaux, dans les prairies et sous le feuillage des hêtres. J'en ai voulu asseoir sur le rivage de la mer, au pied des rochers, à l'ombre des cocotiers, des bananiers et des citronniers en fleurs. (Saint-Pierre, 1999 [1788], p. 93)

3 Mais ce possible ravissement de l'ailleurs sous la plume parfois séductrice du romancier ne saurait expliquer l'extraordinaire succès que Paul et Virginie rencontra durant un long XIX ${ }^{e}$ siècle (éditions, traductions, adaptations, influences littéraires, iconographie, mode des prénoms, etc.). 


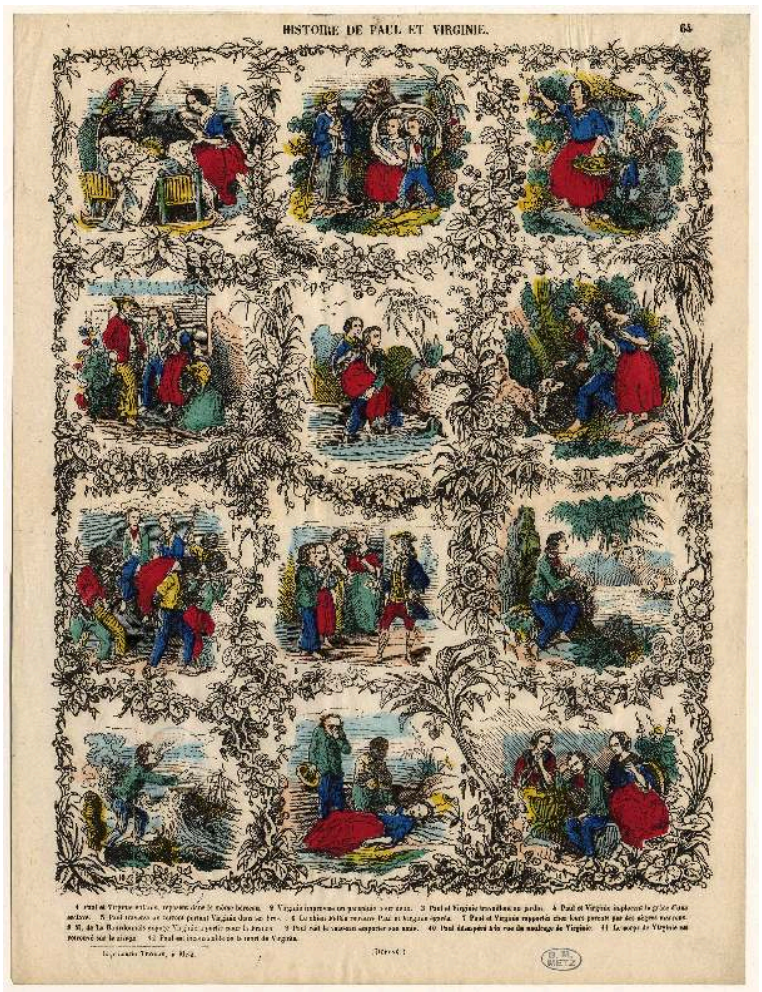

4 Ce best-seller (Toinet, 1969) fut donc aussi, en quelque façon, un mythe culturel, plus exactement le symptôme d'une crise dans la conscience européenne : le révélateur d'un désenchantement de la culture moderne ${ }^{1}$. En effet, cette histoire est une robinsonnade tragique, qui commence sur une note sinistre - «Sur le côté oriental de la montagne qui s'élève derrière le Port-Louis de l'île de France, on voit, dans un terrain jadis cultivé, les ruines de deux petites cabanes [...] ( (Saint-Pierre (1999) [1788], p. 113) et se clôt non moins tristement - «En disant ces mots ce bon vieillard s'éloigna en versant des larmes, et les miennes avoient coulé plus d'une fois pendant ce funeste récit » (ibid., p. 266).

5 Les heurs et malheurs qui rythment successivement le récit ont leurs origines dans une double mise en cause anthropologique. Un déni croisé de civilisation d'abord :

[la vieille et riche tante parisienne, aux portes de la mort] - Mademoiselle, souvenez-vous que vous êtes Française, et que vous devez oublier le pays des sauvages [...]. C'est ce pays-ci qui est pour moi un pays de sauvages [Virginie transplantée à Paris pour faire ses études et devenir une digne héritière]. (ibid., p. 205-206)

Un refus lyrique de la culture écrite ensuite :

Paul. - Oh ! Celle [Virginie] qui a planté ce papayer a fait aux habitants de ces forêts un présent plus utile et plus doux que si elle leur avait donné une bibliothèque. Et en même temps il saisit cet arbre dans ses bras, et le baisa avec transport. (ibid., p. 220)

Le point de vue dominant du texte (son idéologie si l'on veut) consiste à renverser les termes européo-centrés du problème: les couples sauvage/civilisé et culture orale/ culture écrite sont en effet distribués selon un appariement inédit puisque tous les bonheurs seraient dans l'oralité (perdue) et toutes les violences dans les univers régis par l'écrit. C'est cette fiction expérimentale et nostalgique que narre le récit ${ }^{2}$. Un récit 
qui nécessairement survalorise l'oralité et instruit le procès systématique des sociétés qui seraient comme fatalement et puissamment « dénaturées » par l'écrit.

\section{Le murmure des palmistes ou l'oralité heureuse}

Paul et Virginie n'avaient ni horloges, ni almanachs, ni livres de chronologie, d'histoire, et de philosophie. Les périodes de leur vie se réglaient sur celles de la nature. Ils connaissaient les heures du jour par l'ombre des arbres ; les saisons, par les temps où ils donnent leurs fleurs ou leurs fruits; et les années, par le nombre de leurs récoltes. (ibid., p. 129)

7 Ainsi le roman imagine un univers d'oralité première où l'absence de mesure écrite du monde, loin d'être tenue pour un manque ou un miséreux état de vie primitive est au contraire une condition sine qua non du bonheur hic et nunc, immédiate présence au monde et immédiate présence du monde :

Ces douces images répandaient les plus grands charmes dans leurs conversations. «Il est temps de dîner, disait Virginie à la famille, les ombres des bananiers sont à leurs pieds »; ou bien : «La nuit s'approche, les tamarins ferment leurs feuilles. Quand viendrez-vous nous voir? lui disaient quelques amies du voisinage. - Aux cannes de sucre, répondait Virginie [...]. (ibid., p. 168-169)

Cette société de la nature - une cosmologie orale et florale - est à l'évidence une société à l'envers de l'idéologie logocentrique et à coup sûr scriptocentrique occidentale moderne (la série de termes privatifs comme analphabète, illettré, inculte, suffirait à rappeler cet impérialisme de l'écrit) ${ }^{3}$. Ce bonheur préhistorique (les historiens et même anthropologues parlèrent longtemps de civilisation sans écriture) conjugue une conception cyclique d'un temps cosmique agro-botanique et une attention subtile et quotidienne aux rythmes concrets de la végétation. Au plus loin donc des conceptions linéaires du temps mécanique et de l'abstraction comptable de la mémoire écrite. Cet âge d'or, cet âge d'oralité suppose au contraire une mémoire orale vive qui fait corps avec une nature à la fois féconde et généreuse, rythmée et nourricière, complice et harmonieuse, ainsi que voisine et imaginaire :

Quand on l'interrogeait sur son âge et sur celui de Paul : « Mon frère, disait-elle, est de l'âge du grand cocotier de la fontaine, et moi de celui du plus petit. Les manguiers ont donné douze fois leurs fruits, et les orangers vingt-quatre fois leurs fleurs depuis que je suis au monde. " Leur vie semblait attachée à celle des arbres comme celle des faunes et des dryades: ils ne connaissaient d'autres époques historiques que celles de la vie de leurs mères, d'autre chronologie que celle de leurs vergers [...]. (ibid., p. 169)

Dans cette expérience ontologique, sociale et imaginaire du monde, le sensible ne s'oppose pas à l'intelligible, et l'intelligible ne suppose pas la médiation de la raison écrite en ses espaces graphiques, au contraire. Cette oralité augurale et inaugurale du monde et du moi dans ce monde est à la fois native et rituelle :

Lorsque Marguerite [la bien nommée] eut mis Paul au monde je [le sage et bienveillant vieillard retiré du monde occidental] lui fis présent d'un coco des Indes qu'on m'avait donné. Elle planta ce fruit sur le bord de cette flaque d'eau, afin que l'arbre qu'il produirait servît un jour d'époque à la naissance de son fils. Madame de la Tour, à son exemple, en planta un autre dans une semblable intention dès qu'elle fut accouchée de Virginie. Il naquit de ces deux fruits deux cocotiers, qui formaient toutes les archives de ces deux familles ; l'un se nommait l'arbre de Paul, et l'autre, l'arbre de Virginie. Ils crûrent tous deux, dans la même proportion que leurs jeunes maîtres, d'une hauteur un peu inégale, mais qui surpassait au bout de douze ans 
celle de leurs cabanes. Déjà ils entrelaçaient leurs palmes, et laissaient pendre leurs

jeunes grappes de cocos au-dessus du bassin de la fontaine. (ibid., p. 156-157) évidemment des imaginaires de l'idéologie (l'enracinement, la sédentarité, l'autochtonie, le continuum nature/culture, etc.) et des imaginaires arboricoles présents dans la langue (un enfant qui pousse bien, un jardin d'enfant, le tuteur d'un enfant, une belle plante, etc.). Mais ici la cosmologie propre du récit consiste surtout à imaginer, non pas un temps biographique inscrit dans la mémoire grise et privée des archives (cette microsociété se rêve sans État, sans bureaucratie, bref: sans papier d'identité ni actes administratifs de naissance et autres écritures), mais un temps biosymbolique à la fois personnel et social, un authentique arbre généalogique loin des arborescences schématiques voire squelettiques d'une culture graphique qui a quelque chose de morne et de végétatif au regard de l'érotisme génésique doux et latent de la filiation et de l'alliance en la prime jeunesse de la culture orale : "Ainsi se passa leur enfance, comme une belle aube qui annonce un plus beau jour» (ibid., p. 131). Cette empoétisation a sa source dans la contiguïté/continuité des physicalités et les affiliations d'intériorités imaginaires qui irriguent en quelque façon la pensée analogique et son ontologie propre (Descola, 2005, p. 280-320), aux antipodes de la pensée analytique qui, elle, est typique de la dé/raison graphique, système formel de disjonctions et d'espacements (Goody, (1978) [1977] ; Privat, 2006, p. 125-130).

Un dernier exemple - parmi tant d'autres - nous conduira cette fois vers une fiction de présent (y compris pour le lecteur coopératif), une jouvence du présent conjugué à une fulgurance du passé, une délicieuse/délictueuse confusion des percepts et des affects, la conjonction érotique des harmonies végétales des corps :

Virginie s'achemine, à la clarté de la lune, vers sa fontaine [...] semée sur ses bords d'herbes aromatiques [...]. Elle se plonge dans son bassin. La fraîcheur ranime ses sens [...]. Elle se rappelle que dans son enfance sa mère et Marguerite s'amusaient à la baigner avec Paul dans ce même lieu [...]. Elle entrevoit dans l'eau, sur ses bras nus et sur son sein, les reflets des deux palmiers [...] qui entrelaçaient au-dessus de sa tête leurs rameaux verts et leurs jeunes cocos. Elle pense à l'amitié de Paul, plus douce que les parfums, plus pure que l'eau des fontaines, plus forte que les palmiers unis ; et elle soupire [...]. Elle court auprès de sa mère [...] voulant lui raconter ses peines [...], mais son cœur oppressé laissa sa langue sans expression [...] ». (SaintPierre (1999) [1788], p. 174-175)

Ce bain nocturne, lunaire et aquatique, parfumé et féminin, presque immobile, presque silencieux, presque solitaire ; ces contacts sensuels avec la matérialité fertile et féconde du monde; ces corps juvéniles et vierges où les charmes esthésiques le disputent aux enchantements esthétiques, bref cette corps/oralité est ensauvagée, à la fois transgressive (une peau végétale et un inceste symbolique avec son frère de lait, son double, son alter ego) et régressive par son attachement ombilical à des univers prélangagiers, au plus loin de la rationalité scripturale.

\section{La violette scabieuse, ou la lettre qui tue}

Virginie va bientôt être chassée de ces oralités symbiotiques et symphoniques, ce vert paradis des amours enfantines... Paul survivra - un temps - la mort dans l'âme :

Paul aperçut le vaisseau qui emmenait Virginie. Il le vit à plus de dix lieues au large comme un point noir au milieu de l'océan [...]. Il s'assit dans ce lieu sauvage, toujours battu des vents, qui y agitent sans cesse les sommets des palmistes et des 
tatamaques. Leur murmure sourd et mugissant ressemble au bruit lointain des orgues, et inspire une profonde mélancolie. (ibid., p. 197-198) terme) :

Un vaisseau arrivé de France apporta à madame de la Tour une lettre de sa tante. La crainte de la mort, sans laquelle les cœurs durs ne seraient jamais sensibles, l'avait frappée [...]. Elle mandait à sa nièce de repasser en France; ou, si sa santé ne lui permettait pas de faire un si long voyage, elle lui enjoignait d'y envoyer Virginie, à laquelle elle destinait une bonne éducation, un parti à la cour, et la donation de tous ses biens. Elle attachait, disait-elle, le retour de ses bontés à l'exécution de ses ordres. À peine cette lettre fut lue dans la famille qu'elle y répandit la consternation. (ibid., p. 180)

On le voit, cette lettre est toute entière écrite sous le signe du malheur et de la mort. Elle est signe de mort. Elle est arrachement à l'oralité native et domestication à venir des pensées sauvages. La violence symbolique de l'écrit est en fait une assignation légale, un assujettissement à sa toute puissance. Cette lettre qui a l'autorité d'un mandement ne saurait rester lettre morte :

Le lendemain, au lever du soleil [...] le Gouverneur entra dans la case où toute la famille était à table [...] : "Vous avez une tante de qualité et fort riche à Paris, qui vous réserve sa fortune, et vous attend auprès d'elle [...]. Au moins, reprit M. de la Bourdonnais, pour mademoiselle votre fille, si jeune et si aimable, vous ne sauriez sans injustice la priver d'une si grande succession. Je ne vous cache pas que votre tante a employé l'autorité pour la faire venir auprès d'elle. Les bureaux m'ont écrit à ce sujet d'user, s'il le fallait, de mon pouvoir [...]. En disant ces mots, il posa sur la table un gros sac de piastres destiné aux préparatifs de voyage de mademoiselle, de la part de sa tante. (ibid., p. 181)

Cette sorte de lettre de cachet qui dépossède le sujet de la libre disposition de son corps manifeste l'empire de l'ordre de l'écrit et l'emprise des forces temporelles et spirituelles qui instituent cet ordre :

Vers le soir, comme elle était seule avec Virginie, il entra chez elle un grand homme vêtu d'une soutane bleue. C'était un ecclésiastique missionnaire de l'île, et confesseur de madame de la Tour et de Virginie. Il était envoyé par le gouverneur. "Vous voilà riches [...]. Jeune demoiselle, il faut obéir à la Providence. C'est un sacrifice, mais c'est l'ordre de Dieu. Votre voyage en France aura une fin heureuse. (ibid., p. 185)

Pour Virginie, cet arraisonnement par le culte et la culture de l'écrit aura de facto une fin malheureuse, tragique même ${ }^{4}$. La France c'est la distance, c'est l'absence, la France c'est sa souffrance (et non plus l'immédiateté jubilatoire de la coprésence) :

Plus d'un an et demi s'était écoulé sans que madame de la Tour eût des nouvelles de sa tante et de sa fille. Enfin elle reçut, par un vaisseau qui allait aux Indes, un paquet, et une lettre écrite de la propre main de Virginie. Malgré la circonspection de son aimable et indulgente fille, elle jugea qu'elle était fort malheureuse.

Je vous ai déjà écrit plusieurs lettres de mon écriture ; et comme je n'en ai pas eu de réponse, j'ai lieu de craindre qu'elles ne vous soient point parvenues [...]. Ma grandtante fut bien surprise à mon arrivée, lorsque m'ayant questionnée sur mes talents, je lui dis que je ne savais ni lire ni écrire [...]. Elle me mit, dès le lendemain, en pension dans une grande abbaye auprès de Paris, où j'ai des maîtres de toute espèce; ils m'enseignent, entre autres choses, l'histoire, la géographie, la grammaire, la mathématique, et à monter à cheval [...]. Elle m'a fait prendre le titre de comtesse ; mais elle m'a fait quitter mon nom de La Tour [...] (ibid., p. 202-203). 
Cette nouvelle identité culturelle est le fruit amer d'une acculturation/déculturation forcée et forcenée (assez peu de romantisme au sens banal du terme dans cette affaire). L'incorporation de la raison graphique est une déraison, une prison (la tante sera décrite comme dégénérée et même dénaturée, comme l'écrit):

N'ayant à mon arrivée ici personne en qui je pusse prendre confiance, je me suis appliquée nuit et jour à apprendre à lire et à écrire [...]. Ma grand-tante m'a interdit toute correspondance au dehors, qui pourrait mettre obstacle aux grandes vues qu'elle a sur moi. Il n'y a qu'elle qui puisse me voir à la grille, ainsi qu'un vieux seigneur de ses amis [...]. (ibid., p. 204).

On le voit par un retournement classique mais subversif des stigmates culturels, dans la France des Lumières ${ }^{5}$, la scripturalité serait l'immoralité, et la moralité l'oralité. C'est ici qu'intervient le schème historique et idéologique du déracinement culturel et la symbolique florale de la transplantation botanique. Virginie, si belle et désirable comme un fruit de l'île de France, dépérit en France, monde à l'envers du monde, désordre de l'âme, de l'esprit et des corps (on retrouverait aisément l'ami Rousseau le botaniste) :

Dans un post-scriptum Virginie recommandait particulièrement à Paul deux espèces de graines : celles de violettes et de scabieuses. La violette, lui mandait-elle, produit une petite fleur d'un violet foncé, qui aime à se cacher sous les buissons ; mais son charmant parfum l'y fait bientôt découvrir. Elle lui enjoignait de la semer sur le bord de la fontaine, au pied de son cocotier. La scabieuse, ajoutait-elle, donne une jolie fleur d'un bleu mourant, et à fond noir piqueté de blanc. On la croirait en deuil. On l'appelle aussi, pour cette raison, fleur de veuve. Elle se plaît dans les lieux âpres et battus des vents. Elle le priait de la semer sur le Rocher des adieux. (ibid., p. 206).

III. 2 Monument à Bernardin de Saint-Pierre. Jardin des Plantes - Paris. Photo : Marie-Christine Vinson

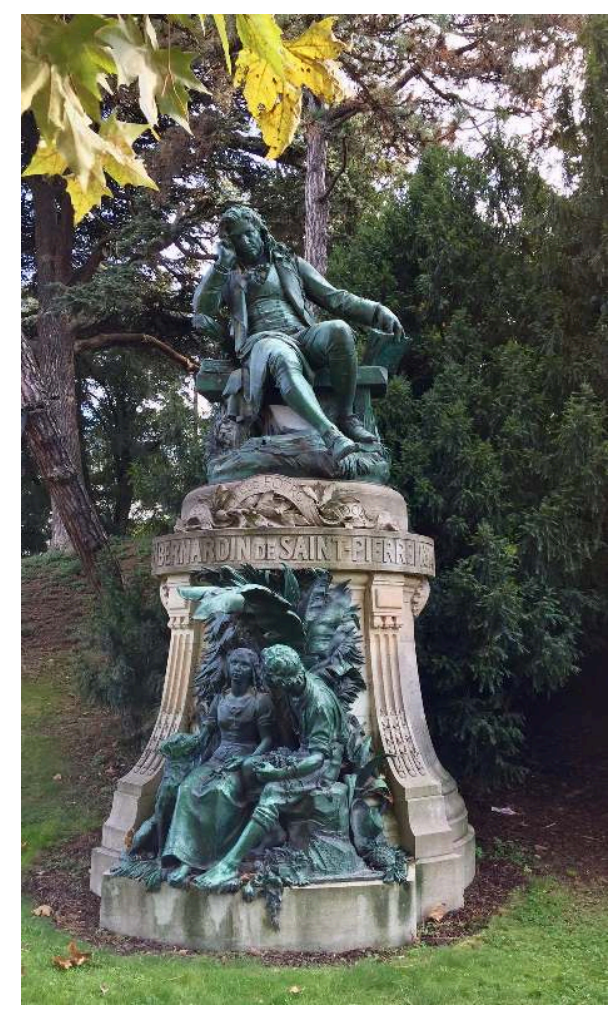


20 C'est ainsi que sous la plume de la virginale Virginie, l'écrit est testament, signe de mort, signature de sa propre mort:

Elle avait renfermé ces semences dans une petite bourse dont le tissu était fort simple, mais qui parut sans prix à Paul lorsqu'il y aperçut un $P$ et un $V$ entrelacés et formés de cheveux, qu'il reconnut à leur beauté pour être ceux de Virginie.

Paul allait rendre le jardin digne d'elle, et y mêler les plantes de l'Europe à celles de l'Afrique, ainsi qu'elle avait entrelacé leurs noms dans son ouvrage. Paul sema avec le plus grand soin les graines européennes, mais il n'en germa qu'un petit nombre, qui ne put venir à sa perfection. (ibid., p. 207-208)

Bref, dans ce jardin de tous les maux, la greffe de l'oralité sur la scripturalité est stérile, comme la vieille tante à Paris, et comme les artificieux Jardins d'Adonis selon Platon $(2007)^{6}$. L'empire de l'écrit sur les pensées ensauvagées est en effet voué à être exclusif, sans compromis, sans concession, sans la moindre sentimentalité, sans espoir véritable de retour non plus :

Un matin, au point du jour (c'était le 24 décembre 1744), Paul, en se levant, aperçut un pavillon blanc arboré sur la montagne de la Découverte. Ce pavillon était le signalement d'un vaisseau qu'on voyait en mer [...]. Le pilote remit au gouverneur les lettres que ce vaisseau apportait de France. Il y en avait une pour madame de la Tour, de l'écriture de Virginie. Paul s'en saisit aussitôt, la baisa avec transport, la mit dans son sein, et courut à l'habitation [...] et aussitôt tout le monde se rassembla chez madame de la Tour pour en entendre la lecture. Virginie mandait à sa mère qu'elle avait éprouvé beaucoup de mauvais procédés de la part de sa grand-tante, qui l'avait voulu marier malgré elle, ensuite déshéritée, et enfin renvoyée dans un temps qui ne lui permettait d'arriver à l'Ile de France que dans la saison des ouragans [...]. À peine cette lettre fut lue que toute la famille, transportée de joie, s'écria : «Virginie est arrivée ! (Saint-Pierre (1999) [1788], p. 231-232).

C'est alors que le récit tourne au tragique (le fameux naufrage, la fin apocalyptique d'un monde où le Jour de la Nativité est Jour des morts). Ainsi le narrateur laisse à entendre combien la culture officielle est, à la fois suffisante et insuffisante, imprévoyante et sourde aux harmonies ou aux disharmonies de la Nature :

M. de la Bourdonnais fit allumer de grands feux de distance en distance sur la grève, et envoya chez tous les habitants du voisinage chercher des vivres, des planches, des câbles, et des tonneaux vides [...]. Un des plus anciens de ces habitants s'approcha du gouverneur, et lui dit: "Monsieur, on a entendu toute la nuit des bruits sourds dans la montagne; dans les bois les feuilles des arbres remuent sans qu'il fasse de vent; les oiseaux de marine se réfugient à terre : certainement tous ces signes annoncent un ouragan. - Eh bien! mes amis, répondit le gouverneur, nous y sommes préparés, et sûrement le vaisseau l'est aussi. En effet tout présageait l'arrivée prochaine d'un ouragan. (ibid., p. 237-238)

23 En effet, le présage, la mantique florale - sur cet îlot de France comme les feuilles des chênes antiques dans la forêt de Dodone - dit le Destin (et peut-être les moyens de s'en protéger) à condition de savoir sinon lire du moins percevoir et interpréter dans la Nature les intersignes (les signes prémonitoires) de la vie et de la mort. Dans une ultime mais illusoire résistance, il reste à s'abandonner aux songes qui sont mensonges, à jouir du mentir-vrai d'un monde imaginaire :

«Marguerite me [le bon vieillard, mémoire orale de l'ensemble du récit] dit : «ô mon bon voisin! Il m'a semblé cette nuit voir Virginie vêtue de blanc, au milieu de bocages et de jardins délicieux. Elle m'a dit: «Je jouis d'un bonheur digne d'envie. Ensuite elle s'est approchée de Paul d'un air riant, et l'a enlevé avec elle. » Mais ce que je trouve encore plus étrange, c'est que madame de la Tour a fait cette même nuit un songe accompagné des mêmes circonstances. Je n'avais jamais remarqué 
dans ces deux dames aucun penchant à la superstition; je fus donc frappé de la concordance de leur songe [...]» (ibid., p. 260-261). monde de Virginie. L'européocentrisme de cette rêverie anthropologique est bien à rebours de l'idéologie modernes des Lumières et du progrès qui a le culte du logos triomphant des superstitions et autres ensauvagements de la pensée orale, exotique et lointaine, ou locale et indigène, archaïque et populaire ${ }^{7}$. Cette raison écrite est déraison dans l'expérience fictive que scénarise ce roman et dans son émouvante dramaturgie d'une mutation anthropologique incisive et décisive (Furet \& Ozouf, 1977, p. 349-369).

\section{Une durable nostalgie culturelle}

Il ne reste alors à la littérature (écrite) qu'à faire contre mauvaise fortune bon style. Cigît l'oralité rêvée selon le roman, tout roman moderne plus ou moins d'ailleurs. Comme si la littérature était un des nouveaux mythes de la modernité vouée à explorer peu ou prou, entre romance et fulgurances, ces "vérités négatives» dont parlait C. LéviStrauss ${ }^{8}$ ou ce paradigme des derniers qui fascine tant et l'ethnographe et l'écrivain (Fabre, 2010, p. 38-65). Les subtiles et innombrables harmonies de la Nature (SaintPierre, 1840 [1836], p. 42-377) sont défaites, mais l'art littéraire en sa cosmographie propre refait un monde souvent nostalgique d'un monde perdu, plus ou moins imaginaire ou utopique, entre la noblesse funèbre d'un memento mori et l'ambiguïté païenne d'un Et in Arcadia ego chez Bernardin" ("moi aussi j'ai vécu en Arcadie mythique, au Royaume d'Oralie " pourraient penser le narrateur et le lecteur) :

On a mis auprès de Virginie, au pied des mêmes roseaux, son ami Paul, et autour d'eux leurs tendres mères et leurs fidèles serviteurs. On n'a point élevé de marbres sur leurs humbles tertres, ni gravé d'inscriptions à leurs vertus ; mais leur mémoire est restée ineffaçable dans le cœur de ceux qu'ils ont obligés [...]. La voix du peuple, qui se tait sur les monuments élevés à la gloire des rois, a donné à quelques parties de cette île des noms qui éterniseront la perte de Virginie [...]. Voici devant nous, au bout de ce vallon, La baie du Tombeau [...] sur les mêmes rivages qu'elle avait honorés de son innocence. (Saint-Pierre (1999) [1788], p. 265-266)

Il reste encore, pour l'ensauvagement symbolique propre à la littérature, d'être une réserve d'altérité culturelle qui trouve toute sa puissance de séduction ou de subversion dans un écho d'oralité, une écho-graphie. Chez notre écrivain, la palme d'oralité - si je peux risquer ce jeu de mots - est dans la quête d'une harmonie fusionnelle entre une nature anthropique (elle parle, elle murmure tendrement) et d'une humanité naturée (elle est toute sève). Paul et Virginie vivent en effet dans les plantes et des plantes (fruits et légumes). Ils sèment et s'aiment. Ils cueillent et recueillent. Ils pêchent sur le rivage généreux (Paul nage comme un poisson), mais ne sauraient chasser. Ils ne tuent point. Ils ne versent pas le sang. Ils sont non violents et sans commerce ni monnaie autre que le commerce d'un entre soi affectueux et d'un échange probe en toute circonstance. La violence c'est l'altérité tribale et scribale. Or, justement cet écho qui a tant fasciné Bernardin est comme une résonnance sonore, musicale, vocale, acousmatique, narcissique, mythologique parfois telle qu'à l'aube des temps; bref une oralité pure et subtile, une harmonie oraculaire strictement sans équivalent à l'écrit, une choralité magique qui s'enchante de la présence d'une absente ${ }^{10}$ et non de sa seule trace (écrite), un lointain soudain si proche, un opéra 
fabuleux multi-sensoriel, très provisoirement protégé des menaces de la violence de la lettre :

À l'entrée de ce bassin [...], les échos de la montagne répètent sans cesse le bruit des vents qui agitent les forêts voisines, et le fracas des vagues qui brisent au loin sur les récifs. Au pied des cabanes on n'entend plus aucun bruit [...]. Un grand silence règne [...]. Tout est paisible, l'air, les eaux et la lumière. À peine l'écho y répète le murmure des palmistes qui croissent sur leurs plateaux élevés, et dont on voit les longues flèches toujours balancées par les vents [...] - Quand l'écho me [Virginie] fait entendre les airs que tu [Paul] joues sur ta flûte, au haut de la montagne, j'en répète les paroles au fond de ce vallon [...] - La cascade voisine, le papayer qu'elle avait planté, les pelouses où elle aimait à courir, les carrefours de la forêt où elle se plaisait à chanter, firent tour à tour couler ses larmes [Paul]; et les mêmes échos, qui avaient retenti tant de fois de leurs cris de joie communs, ne répétaient plus maintenant que ces mots douloureux : «Virginie ! ô ma chère Virginie ! (ibid., p. 115, 172, 250).

Ce lamento poétique et cette incantation vocale aux charmes parfois désuets de la prose bernardine nous toucheront longtemps encore comme la plainte stylisée d'une oralité de fiction. Aujourd'hui par contre, plus vivace que jamais, demeure le mythe publicitaire d'un monde exotique et insulaire vierge d'écrit(s), tant reste puissant dans les cultures contemporaines le désir d'une oralité originelle enfin retrouvée.

III. 3 « Votre cœur est déjà là-bas ». Publicité Air Mauritius.

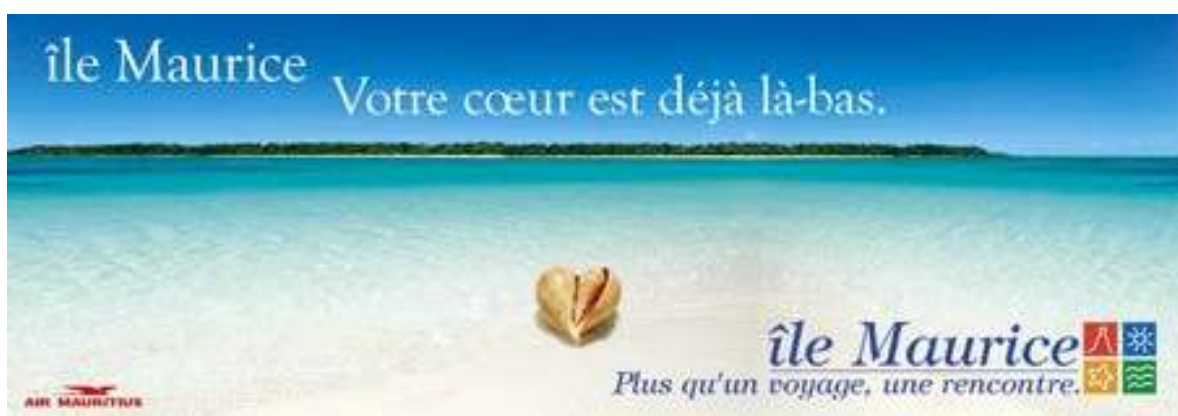

\section{BIBLIOGRAPHIE}

CERTEAU, M. (DE) (1975). « Une France sauvage ». In : Certeau, M. (de). Une politique de la langue. La Révolution française et les patois. Paris : Gallimard, p. 136-154.

CONDORCET, N. (DE) (1989) [1791]. « Un dictionnaire, un journal, un almanach ». In : Coutel, C. \& Kinzler, C. (éds). Cinq mémoires sur l'instruction publique. Paris : Édilig. p. 163-167.

DESCOLA, P. (2005). « Les vertiges de l'analogie ». In : Par-delà nature et culture. Paris : Gallimard, p. 280-320.

FABRE, D. (2010). « D'une ethnologie romantique ». In : Fabre D. \& Privat J.-M. (dirs), Savoirs romantiques. Une naissance de l'ethnologie. Nancy : Presses universitaires de Nancy, p. 38-65. 
FURET, F. \& OZOUF, J. (1977). « Trois siècles de métissage culturel ». In : Lire et écrire. L'alphabétisation des Français de Calvin à Jules Ferry, I. Paris : Éditions de Minuit. p. 349-369.

GOODY, J. (1978) [1977]. La Raison graphique. La domestication de la pensée sauvage. Trad. de l'anglais par J. Bazin \& A. Bensa. Paris : Éditions de Minuit.

FLAUBERT, G. (1971) [1857]. Madame Bovary. Paris : Garnier Frères.

LAMARTine, A. (de) (1979) [1849]. Graziella. In : Gardair, J.-M. (éd.). Paris : Gallimard.

LÉVI-STRAUSS, C. (1958). « Le critère de l'authenticité ». In : Anthropologie structurale. Paris : Plon. p. 425-428.

LÉVI-STRAUSS, C. (1973) [1958]. « Mythologie et ritualité. La geste d'Asdiwal ». In : Anthropologie structurale, 2. Paris : Plon. p. 175-233.

Platon (2007). « Les jardins d'Adonis ». In : Phèdre. Paris : Librairie générale française, 276b-c, p. 306-307.

PRIVAT, J.-M. (2006). « Un habitus littératien? ». Pratiques, 131-132. p. 125-130.

SAINT-PIERRE, B. (de) (1773). Voyage à l'Isle de France à l'isle de Bourbon, au Cap de Bonne-Espérance, etc. Avec des observations nouvelles sur la nature et sur les hommes. Amsterdam : Merlin. En ligne :

https://gallica.bnf.fr/ark:/12148/bpt6k1019923.texteImage.

SAINT-PIERRE, B. (de) (1999) [1788]. Paul et Virginie. Paris : Librairie générale française.

SAINT-PIERRE, B. (de) (1840) [1836]. « Harmonies de la Nature ». In : Aimé-Martin, L. (éd.). CEuvres

posthumes, I. Paris : Ledentu. p. 42-631.

\section{NOTES}

1. Rappelons-nous dans Graziella la belle et terrible expression de Lamartine (1979 [1849], p. 96, 99, 134) quand il évoque la violence du déchirement culturel de la jeune héroïne arrachée manu militari à ses oralités enfantines et amoureuses: "Nous essayâmes [...], un soir de leur lire Paul et Virginie [...]. Quand je fus arrivé au moment où Virginie, rappelée en France par sa tante, sent, pour ainsi dire, le déchirement de son être en deux [c'est nous qui soulignons]. [...] La poésie n'a pas d'écho plus sonore et plus prolongé que le cœur de la jeunesse où l'amour va naître. »

2. L'auteur a visité - à pied - l'île en 1773 et a rapporté une relation de ses observations - Voyage à l'Isle de France... d'un réel intérêt documentaire, notamment en matière de description de la flore et de la faune. On sait aussi que l'écrivain fut assez vite désillusionné par ces tristes tropiques; il s'indigna aussi de la situation faite aux « nègres » et argumenta contre l'esclavage (Saint-Pierre, 1773).

3. On se souvient de la réserve critique de C. Lévi-Strauss (1958, p. 426) sur l'arasement des relations interpersonnelles directes et l'amenuisement d'un rapport au monde concret dans les cultures de l'écrit: « Je n'entends pas me livrer au paradoxe et définir de façon négative l'immense révolution introduite par l'invention de l'écriture. Mais il est indispensable de se rendre compte qu'elle a retiré à l'humanité quelque chose d'essentiel, en même temps qu'elle lui apportait tant de bienfaits ».

4. Les exemples de micro-résistances factuelles à l'écrit sont très nombreux: "De temps en temps madame de la Tour lisait publiquement quelque histoire touchante de 
l'Ancien ou du Nouveau Testament. Ils raisonnaient peu sur ces livres sacrés; car leur théologie était toute en sentiment [...] » (Saint-Pierre (1999) [1788], p. 160). Les contes eux-mêmes avertissent des périls de l'écrit, ses lieux dangereux ou ses ambitions funestes : «La nuit venue, ils soupaient à la lueur d'une lampe; ensuite madame de la Tour ou Marguerite racontait quelques histoires de voyageurs égarés la nuit dans les bois de l'Europe infestés de voleurs, ou le naufrage de quelque vaisseau jeté par la tempête sur les rochers d'une île déserte » (160).

5. Songeons, par exemple, à l'abbé Grégoire et à l'extinction des oralités patoisantes (Certeau, 1975, p. 136-154); à l'éloge de l'imprimerie par Condorcet et à son plan d'études (Condorcet, 1989 [1791], p. 173-174, 262-268); aux travaux des naturalistes eux-mêmes (Linné, Buffon, Saint-Pierre lui-même ; voir Ill. 2) ou encore à ce monument de mise en ordre écrite et graphique du monde qu'est par excellence l'Encyclopédie ou Dictionnaire raisonné des sciences, des arts et des métiers, par une société de gens de lettres, mise en ordre et publié par M. Diderot [...] (1751-1772) .

6. «Socrate : Le paysan intelligent qui prend soin de ses semences et qui veut qu'elles donnent des fruits, est-ce que ce paysan les sèmerait l'été dans les jardins d'Adonis et qu'il se réjouirait en voyant ces fruits devenir beaux en huit jours? [...]. Ce serait comme écrire sur l'eau et semer au moyen d'une plume trempée dans l'encre noire avec des discours inertes incapables d'enseigner vraiment la vérité ». (Platon, 2007, 276b-c)

7. Si l'on convient de désigner d'un point de vue jacobin et éclairé les paysans comme les "sauvages du dedans", se dessine alors une homologie de situation (historique et culturelle) entre les «naturels » d'ici et les «naturels » de là-bas. Il est remarquable que dans cette idéologie la campagne est marquée «soit comme une origine (une contre-société idyllique), soit perçue comme le résidu d'un développement inachevé (une sauvagerie) [...]. Au projet ethnographique se substitue une réduction anthropologique, et l'on se place désormais au niveau des sciences naturelles [...]. On peut décrire l'homme de la campagne comme une plante ou une roche. La référence naturaliste devient ainsi le moyen d'une disqualification, l'affirmation d'une minorité. Elle justifie et appelle l'intervention extérieure », (Certeau, 1975, p. 144-148). Et le roman ne serait qu'apparemment exotique.

8. «Les institutions décrites par le mythe peuvent être inverses des institutions réelles. C'est le cas quand le mythe sert à exprimer une vérité négative [...], les virtualités latentes ou inconscientes d'une culture [...], les possibilités inhérentes à sa structure. Il cherche non à peindre le réel, mais à justifier la côte mal taillée en quoi il consiste, puisque les positions extrêmes y sont imaginées, seulement pour les démontrer intenables », (Lévi-Strauss, 1973 [1958], p. 208-209).

9. Le vieux sage qui écrit du latin (langue morte) sur les arbres: "L'idée me vint de graver une inscription sur la tige de ce roseau [...]. J'ai tant de plaisir à lire une inscription bien faite ; il me semble alors qu'une voix humaine sorte de la pierre [...] » (Saint-Pierre (1999) [1788], p. 153).

10. Paul « se mit à table avec nous auprès de la place où se mettait la compagne de son enfance; et, comme si elle l'eût encore occupée, il lui adressait la parole et lui présentait les mets qu'il savait lui être les plus agréables ; mais dès qu'il s'apercevait de son erreur il se mettait à pleurer » (Saint-Pierre (1999) [1788], p. 199). 


\section{RÉSUMÉS}

Le roman de Bernardin de Saint-Pierre, Paul et Virginie (1999 [1788]), se donne à lire comme le récit d'un affrontement tragique entre deux univers: le monde des oralités premières et heureuses; le monde des écrits lointains, répressifs ou dépressifs. Ainsi le récit se présente comme la mise à l'épreuve expérimentale de deux cosmologies contradictoires et comme le symptôme d'une crise dans la conscience européenne. Le texte dénonce à sa façon, ce désenchantement du monde - tous les personnages meurent... - et énonce une forme d'utopie poétique où l'écho par exemple - un écho graphique d'oralité à vrai dire -participerait d'un opéra fabuleux imaginaire.

The novel by Bernardin de Saint-Pierre, Paul et Virginie (1999 [1788]), can be read as the story of a tragic confrontation between two universes: the world of happy and primary oralities; the world of distant, repressive or depressive writings. Thus, the narrative can be read as a testing of two contradictory cosmologies and as a symptom of a crisis in European consciousness. The text denounces in its own way, this loss of the magic quality of the world -all the characters die...and gives expression to a form of poetic utopia in which the echo, for example -actually a graphic echo of orality- would be part of a fabulous opera of the imagination.

\section{INDEX}

Keywords : Paul et Virginie, ethnocriticism, primary orality, insularity, Europe, writing, symbolic violence, resistance to writing, poetry of the echo, cultural nostalgia

Mots-clés : Paul et Virginie, ethnocritique, oralité première, insularité, Europe, littératie, violence symbolique, résistance à l'écrit, poésie de l'écho, nostalgie culturelle

\section{AUTEUR}

\section{JEAN-MARIE PRIVAT}

Université de Lorraine, Crem, F-57000 Metz, France 